\title{
Test yourself answer: multiple firm soft tissue masses
}

\author{
Anika Choraria $^{1}$ (D) Asif Saifuddin ${ }^{1}$
}

Received: 3 February 2021 / Revised: 15 March 2021 / Accepted: 16 March 2021 / Published online: 26 March 2021

(C) ISS 2021

\section{Diagnosis}

\section{Multifocal tumoral calcinosis}

\section{Discussion}

Whole body bone scintigraphy demonstrates intense increased radionuclide uptake at the left shoulder and left groin, with faint uptake in relation to the right greater trochanter (Fig. 1). The pelvic radiograph shows a focus of amorphous lobular calcification overlying the left obturator foramen and a further deposit in the right trochanteric region, corresponding to the areas of increased uptake on bone scintigraphy (Fig. 2). MRI of the pelvis shows two well-defined lobular masses which demonstrate heterogeneous low signal intensity on T1- and T2-weighted sequences in line with the diffuse calcified nature of the masses seen on the radiograph. One is located anterior to the left pectineus muscle without deeper extension into the pelvic cavity, and the other is found adjacent to the right sub-gluteus medius bursa (Fig. 3). MRI of the left shoulder demonstrates a mass located in the subacromial-subdeltoid bursal region displaying similar signal characteristics (Fig. 4). Regarding all the lesions, there is no adjacent soft tissue or osseous abnormality. A CT-guided biopsy of the left groin mass yielded purely calcific material with no evidence of malignant cells. A diagnosis of multifocal tumoral calcinosis was made.

Tumoral calcinosis involves the accumulation of calcium hydroxyapatite crystals typically in a peri-articular location in the anatomic distribution of a bursa $[1,2]$. The calcium

The case presentation can be found at doi: https://doi.org/10.1007/ s00256-021-03761-4)

Anika Choraria

choraria.anika@gmail.com

1 Department of Radiology, Royal National Orthopaedic Hospital, Brockley Hill, Stanmore, Middlesex HA7 4LP, UK deposition results in a foreign body granulomatous reaction with the formation of soft tissue masses comprised of fluidfilled cystic spaces, oedema and fibrosis [3]. The most classical sites of involvement are the hip, followed by the elbow and shoulder, with unifocal lesions predominating [3]. Smack et al. classified cases into three types: (1) primary normal phosphataemic tumoral calcinosis, (2) primary hyperphosphataemic tumoral calcinosis, and (3) secondary tumoral calcinosis [4]. The primary types typically present within the first two decades of life with no specific gender predilection, although a higher incidence is noted in patients of African ethnicity [2]. The secondary type is commonly seen in patients with chronic renal failure and secondary hyperparathyroidism [3]. Lesions in tumoral calcinosis can have varying imaging appearances. Typically, MRI demonstrates lobulated masses with low signal intensity on all pulse sequences due to the calcium content of lesions [5]. Increased signal intensity on T1W may be seen due to fatty tissue or haemorrhage, while blood-filled cystic spaces may be hyperintense on $\mathrm{T} 2 \mathrm{~W}$. Fluid-calcium levels may be present and post-contrast images show enhancement within the cyst walls [3].

Several differential diagnoses can be considered. The soft tissue calcifications related to connective tissue diseases display characteristic diffuse sheet-like deposits in the muscles (calcinosis universalis) and are typically located in the subcutaneous tissues rather than in a peri-bursal location (calcinosis circumscripta), making these conditions distinct from tumoral calcinosis at imaging $[6,7]$. Location of the lesion is a useful differentiator - as the names suggest, calcific tendonitis is centred on a tendon and calcific myonecrosis usually follows from a history of trauma and is intramuscular [6]. Synovial chondromatosis is usually distinguishable by demonstrating both 'rings-and-arcs' morphology of the calcifications and an intraarticular location [8]. Involvement of bone or calcification only involving a portion of the tumour can make osteosarcoma or synovial sarcoma distinct from tumoral calcinosis at imaging [9]. Myositis ossificans can be radiographically differentiated by a clinical history of trauma, its pattern of mineralisation and lack of lobular morphology and predilection for different anatomical locations [6]. 
In our patient, presentation was late in adulthood with multifocal calcified soft tissue lesions, normal serum biochemistry, no active underlying condition and no relevant family history. To the best of our knowledge, a case of primary multifocal tumoral calcinosis in an adult with normal blood results has not been reported previously. The treatment of choice of symptomatic tumoral calcinosis lesions is surgical resection, but there is a risk of recurrence [6]. Phosphate deprivation has also had variable success rates in both normo- and hyperphosphataemic cases [10]. After a period of clinical and radiological follow-up, our patient remained asymptomatic so no surgical intervention was carried out, and a conservative management approach was followed.

The identification of a calcified soft tissue mass on imaging should prompt careful clinical and biochemical correlation given that a number of pathologies can be responsible. The radiologist plays a key role in guiding the clinical team to either performing invasive procedures for histological confirmation in atypical cases or avoiding such unnecessary procedures and instead selecting suitable interval follow-up imaging to reach a definitive diagnosis.

\section{Declarations}

Conflict of interest The authors declare no competing interests.

\section{References}

1. Inclan A, Leon P, Camejo MG. Tumoral calcinosis. J Am Med Assoc. 1943;121(7):490-5.

2. Harkess JW, Peters HJ. Tumoral calcinosis. A report of six cases. J Bone Joint Surg Am. 1967;49(4):721-31.

3. Martinez S. Tumoral calcinosis: 12 years later. Semin Musculoskelet Radiol. 2002;6(4):331-9.

4. Smack D, Norton SA, Fitzpatrick JE. Proposal for a pathogenesisbased classification of tumoral calcinosis. Int J Dermatol. 1996;35(4):265-71.

5. Sayar I, Peker K, Kapısız A, Bostancı IE, Gürbüzel M, Isik A, et al. Multifocal tumoral calcinosis in a 4-year-old girl. Am J Case Rep. 2014;15:103-6.

6. Olsen KM, Chew FS. Tumoral calcinosis: pearls, polemics, and alternative possibilities. RadioGraphics. 2006;26(3):871-85.

7. Bittmann S, Günther MW, Ulus H. Tumoral calcinosis of the gluteal region in a child: case report with overview of different softtissue calcifications. J Pediatr Surg. 2003;38(8):E4-7.

8. Kransdorf MJ, Meis JM. From the archives of the AFIP. Extraskeletal osseous and cartilaginous tumors of the extremities. Radiogr Rev Publ Radiol Soc N Am Inc. 1993;13(4):853-84.

9. Jones BC, Sundaram M, Kransdorf MJ. Synovial sarcoma: MR imaging findings in 34 patients. AJR Am J Roentgenol. 1993;161(4):827-30.

10. Davies M, Clements MR, Mawer EB, Freemont AJ. Tumoral calcinosis: clinical and metabolic response to phosphorus deprivation. Q J Med. 1987;63(242):493-503.

Publisher's note Springer Nature remains neutral with regard to jurisdictional claims in published maps and institutional affiliations. 